\title{
Effect of Environmental Conditions on the Concentration of Tear Inflammatory Mediators During Contact Lens Wear
}

\author{
Vicente Martín-Montañez, PhD, *广 Amalia Enríquez-de-Salamanca, PhD, ${ }^{* \dagger}$ \\ Alberto López-de la Rosa, MSc,* Alberto López-Miguel, PhD,* Itziar Fernández, PhD, *† \\ Margarita Calonge, MD, *广 José M. González-Méijome, PhD, ł and María J. González-García, PhD*广
}

\begin{abstract}
Purpose: To analyze the influence of environmental conditions on the concentrations of tear inflammatory mediators during contact lens (CL) wear.
\end{abstract}

\begin{abstract}
Methods: Fifty-four CL wearers completed 4 visits combining the bilateral use of omafilcon A or comfilcon A CL and a 90-minute exposure to 2 environmental conditions: standard [50\% relative humidity $(\mathrm{RH}), 23^{\circ} \mathrm{C}, 930 \mathrm{mb}$ ] or adverse $(5 \% \mathrm{RH}$, localized air flow, $23^{\circ} \mathrm{C}, 750 \mathrm{mb}$ ). Four microliters of tears was collected by capillarity from each subject. Changes in concentration of epidermal growth factor (EGF); interleukin (IL)-1 receptor antagonist, IL-1 $\beta$, IL-2, IL-4, IL-6, and IL-8; tumor necrosis factor (TNF) $\alpha$; monocyte chemoattractant protein-1; and matrix metalloproteinase (MMP)-9 were analyzed. The effects of the environment, CL type, and symptoms were evaluated using a 3-way mixed analysis of variance with repeated measures.
\end{abstract}

Results: Under the standard condition, EGF significantly increased [0.36; $95 \%$ confidence interval $(\mathrm{CI}), 0.08$ to 0.64$]$, and IL-1 $\beta(-0.48$; $95 \% \mathrm{CI},-0.84$ to -0.12$)$ and IL-2 $(-0.48 ; 95 \% \mathrm{CI},-0.87$ to -0.09$)$ significantly decreased. Under the adverse condition, IL-6 significantly increased $(0.35 ; 95 \% \mathrm{CI}, 0.09$ to 0.62$)$. Comparing conditions, EGF change was significantly lower $(P=0.02)$ and IL-1 $\beta$, IL-2, IL-6, and TNF- $\alpha$ changes were significantly higher $(P \leq 0.04)$ under the adverse condition. Additionally, IL-1 $\beta$ significantly decreased with comfilcon A $(-0.51 ; 95 \% \mathrm{CI},-0.88$ to -0.15$)$, being significantly lower $(P=0.01)$ than the change with omafilcon A $(0.06 ; 95 \% \mathrm{CI},-0.23$ to 0.35$)$.

Conclusions: The secretion of several tear inflammatory mediators during CL wear differs depending on the environmental conditions and the CL type used. These outcomes might help to understand the

Received for publication July 23, 2015; revision received May 17, 2016; accepted June 5, 2016. Published online ahead of print July 20, 2016.

From the *IOBA, University of Valladolid, Valladolid, Spain; $†$ Networking Research Center on Bioengineering, Biomaterials and Nanomedicine (CIBERBBN), Valladolid, Spain; and †Clinical and Experimental Optometry Research Laboratory, Center of Physics, University of Minho, Braga, Portugal.

Partially supported by the Junta de Castilla y León and the European Social Fund through Project VA317-11 (scholarship).

The authors have no conflicts of interest to disclose.

Presented in part at The British Contact Lens Association 2015 Clinical Conference and Exhibition, May 2015, Liverpool, United Kingdom.

Supplemental digital content is available for this article. Direct URL citations appear in the printed text and are provided in the HTML and PDF versions of this article on the journal's Web site (www.corneajrnl.com).

Reprints: María J. González-García, PhD, IOBA, University of Valladolid, Paseo de Belén, 17, 47011 Valladolid, Spain (e-mail: mjgonzalez@ioba.med.uva.es).

Copyright $(2016$ Wolters Kluwer Health, Inc. All rights reserved. effect of the environment and CL materials on the ocular surface of CL wearers.

Key Words: cytokines, chemokines, inflammation, environment, contact lens

(Cornea 2016;35:1192-1198)

C ontact lens (CL) wearers are daily exposed to artificially controlled environments such as air-conditioned offices, buildings, or aircraft cabins. These indoor spaces involve challenging environmental conditions such as low relative humidity (RH), air flow, and/or decreased barometric pressure. Airplane cabins in particular, because of their characteristics regarding $\mathrm{RH}$, air renewal, etc., represent an adverse environment, which combines these conditions all together. ${ }^{1}$ Previous studies have shown that adverse conditions elicit the development of dryness symptoms in dry eye patients and CL wearers. ${ }^{2-8}$ These desiccating scenarios also have a negative impact on clinical signs of the ocular surface, ${ }^{2,9-11}$ including a decrease in tear volume and stability and an increase in conjunctival hyperemia and corneal staining. As the presence of a CL on the ocular surface reduces tear film stability, ${ }^{12} \mathrm{CL}$ wear in adverse environments is likely to exacerbate further these ocular symptoms.

It is known that some CL-related complications are somewhat mediated through the release of tear inflammatory mediators, such as giant papillary conjunctivitis, which presents altered tear levels of eotaxin, ${ }^{13}$ or corneal neovascularization, which is mediated by vascular endothelial growth factor. ${ }^{14}$ Likewise, the concentration of several tear molecules, such as interleukin (IL)-6, IL-8, and epidermal growth factor (EGF), ${ }^{15-18}$ has been shown to be upregulated in CL wearers. Moreover, CL type could be key in this, as differences have been found between hard and soft CL wearers. ${ }^{16,19}$ These findings suggest that there may be an underlying inflammatory process related to $\mathrm{CL}$ wear. The expression of some tear proteins has also been found altered in subjects with CL-related discomfort ${ }^{20}$; this discomfort can lead to decreased wearing time and discontinuation of CL wear. ${ }^{21}$

Exposure to desiccating environments exacerbates dry eye clinical signs ${ }^{2,9,10}$ and increases certain proinflammatory molecules regardless of the presence of dry eye disease. ${ }^{10}$ We hypothesized that transient exposure to an artificially adverse environment could also elicit the expression of some 
proinflammatory mediators in CL wearers. With that in mind, the purpose of the study was to analyze the influence of controlled environmental conditions, CL type, and CL-related symptoms on the concentration of tear inflammatory mediators in CL wearers.

\section{MATERIALS AND METHODS}

\section{Ethical Issues}

The study complied with the tenets of the Declaration of Helsinki, and the protocol was approved by the University of Valladolid Ethics Committee. The nature of the research and protocols was explained to the subjects before written informed consent was obtained during the preliminary visit.

\section{Participants}

Fifty-four soft CL wearers [20 men and 34 women; mean age \pm standard deviation $(\mathrm{SD}), 20.0 \pm 7.3$ years; range, 18-45] were included. The criteria for selecting subjects were age between 18 and 45 years, a myopic spherical equivalent between -1.00 and -5.00 diopters (D), astigmatism error less than or equal to $0.75 \mathrm{D}$, and having worn CLs for at least the last 6 months before the study. Exclusion criteria were taking systemic or ocular medication (different from artificial tears), ocular abnormalities, and a history of ophthalmic disease or surgery, including refractive procedures.

The ocular surface disease index (OSDI) questionnaire was administered to categorize subjects based on their discomfort with their habitual CL wear (discomfort-based grouping). Those having an OSDI score $<15$ were classified as asymptomatic, and those having a score $\geq 15$ were classified as symptomatic. ${ }^{22}$

At the preliminary visit (V0), all subjects were fitted with the 2 study CLs-omafilcon A and comfilcon A (CooperVision, Irvine, CA) - to confirm that both types of CLs fit each subject properly. These CLs were chosen because of their similarities in CL parameters and physical properties of their CL blister solutions (osmolarity, $\mathrm{pH}$, surface tension, and viscosity). ${ }^{23}$

The right eye was chosen as the study eye for tear molecule analysis, despite the fact that the CLs were worn bilaterally.

\section{Study Protocol}

The recruited subjects were exposed to 2 different environmental conditions wearing each of the 2 CL types for each exposure, making a total of 4 visits by each subject, thus volunteers wore each CL type under 2 different environmental conditions. At the beginning of each visit, subjects were bilaterally fitted with the randomized CL, wore the CLs for 15 minutes, and immediately after performing the tear collection, they were exposed to the randomized environmental condition. The order in which participants were exposed with each CL under each environmental condition was randomized, and visits were spaced between 2 and 5 days. Subjects were instructed not to use their CLs or artificial tears from 24 hours before starting the study until after they had finished all visits.

\section{Environmental Conditions}

Subjects were exposed within an environmental chamber (CERLab, IOBA, University of Valladolid) ${ }^{9}$ to 2 different controlled environmental conditions: (1) "standard condition" $\left(50 \% \mathrm{RH}, 23^{\circ} \mathrm{C}\right.$ temperature, and $930 \mathrm{mb}$ of atmospheric pressure, which is the average pressure in Valladolid, Spain) and (2) "adverse condition" (5\% RH, localized air flowmean air velocity $0.43 \mathrm{~m} / \mathrm{s}, 23^{\circ} \mathrm{C}$ temperature, and $750 \mathrm{mb}$ atmospheric pressure - similar to that typically found within an airplane cabin during flight). ${ }^{24-26}$ Environmental chamber exposure lasted 90 minutes, while the individuals were seated watching a film on a 55-inch television (LG Electronics Inc, Gumi, South Korea).

\section{Tear Sample Collection}

Tear sample collection was performed twice per visit: (1) after wearing the CL for 15 minutes and just before the exposure to each environmental condition (PRE) and (2) immediately after 90 minutes of exposure (POST).

A 4- $\mu \mathrm{L}$ sample of basal tears was collected from the external canthus using a glass capillary tube (Drummond Scientific, Broomall, PA) in a nontraumatic way, trying to avoid reflex tear secretion as much as possible. Tear samples were diluted (1/10) in assay buffer and frozen as described previously. $^{27}$

\section{Analysis of Tear Molecule Concentration}

The concentrations of EGF; IL-1 receptor antagonist (RA); IL-1 $\beta$, IL-2, IL-4, IL-6, IL-8; tumor necrosis factor (TNF) $\alpha$; monocyte chemoattractant protein-1 (MCP-1); and matrix metalloproteinase-9 (MMP-9) were measured simultaneously with a 10-plex immunobead-based assay (10X-plex magnetic human cytokine/MMP-9 panel; Millipore, Billerica, MA) in a Luminex IS-100 instrument (Luminex Corp, Austin, TX). The samples were analyzed according to the manufacturer's procedure as previously described. ${ }^{27}$ The minimum detectable concentrations (in picograms per milliliter) for molecules analyzed were as follows: 1.23 for EGF, IL-1RA, IL-4, IL-8, MCP-1, and MMP-9; 1.18 for TNF- $\alpha$; 1.16 for IL2; 1.12 for IL-6; and 1.05 for IL-1 $\beta$.

In some cases, the assayed molecule was undetectable. Cytokine levels below the limit of detection were imputed using the robust regression on order statistics method introduced by $\mathrm{Helsel}^{28}$ and implemented in the NADA (nondetects and data analysis) package. ${ }^{29}$ However, molecules detected in less than $50 \%$ of the samples in every condition were not analyzed further because the statistical analysis might be biased. ${ }^{30}$

\section{Data Analysis}

The sample size was calculated with the online freeware "Power Analysis for ANOVA Designs". ${ }^{31}$ It took into account that 2 groups and 4 clinical scenarios were compared, level of significance was determined as 0.05 , effect size as $0.40,{ }^{32}$ and statistical power as $80 \%$. Based on these parameters, sample size calculated was 25 subjects for each symptomatic and asymptomatic group. We included 2 additional subjects per 
group in case of dropout; therefore, the total sample size was 27 subjects for each group.

Sample distributions for quantitative variables were analyzed using the Mann-Whitney $U$ test, whereas qualitative variables were compared using either the $\chi^{2}$ test or the Fisher exact test if cell sizes were too small.

Tear molecule analysis data were log-transformed (log 2 ), which normalizes the tear concentration data, before conducting statistical analysis. The change in tear molecule concentration between both moments of environment exposure (PRE and POST) was calculated as the difference with the following formula: change $=\log _{2}\left(\mathrm{Y}_{\text {post }}\right)-\log _{2}\left(\mathrm{Y}_{\mathrm{pre}}\right)$, where " $Y_{\text {post }}$ " is the " $Y$ " tear molecule concentration after the exposure and " $\mathrm{Y}_{\mathrm{pre}}$ " is the "Y" tear molecule concentration before the exposure. The main advantage of this formula is that equal positive and negative change values will be symmetric and reciprocal, that is, a change of -1 means that the amount of the molecule is half of the previous value and a change of +1 means double. The "change" numerical value thus obtained was considered as the main variable. Changes in molecules whose $95 \%$ confidence interval (CI) did not include the " 0 " value were considered statistically significant.

A multivariable analysis, a repeated-measures analysis of variance (ANOVA), was used to analyze the effect of environmental condition (standard and adverse), CL type (omafilcon A and comfilcon A), and discomfort-based grouping (asymptomatic and symptomatic subjects), and their interactions, on the change in tear molecule concentration. Two intrasubject (environmental condition and CL type) and 1 intersubject (discomfort-based grouping) factors, and their interactions, were considered. Pairwise comparisons were based on Student $t$ tests. $P$-values were adjusted for multiple testing by the Holm method. ${ }^{33}$

Statistical analysis was carried out by a licensed statistician (coauthor I. Fernández) using R Statistical Software. ${ }^{34}$ Package $\mathrm{Car}^{35}$ was used to fit repeated-measures ANOVA models. $P$ values less than or equal to 0.05 were considered statistically significant.

\section{RESULTS}

The OSDI-based classification divided the participants into an asymptomatic group (11 males and 16 females; white; mean age $25.8 \pm 6.0$ years) and a symptomatic group (9 males and 18 females; white; mean age $28.8 \pm 8.3$ years). None of the participants suffered from systemic diseases. Clinical variables included at the preliminary visit for both groups are shown in Table 1 . The subject sex and age did not differ significantly $(P=0.573$ and $P=0.215$, respectively) between the groups. Likewise, no significant differences were found between the groups in terms of CL wear time $(P=0.476), 7.1 \pm 5.3$ years for the asymptomatic group and $6.7 \pm 4.5$ years for the symptomatic one. CL wear schedule did not differ between groups either $(P=$ 0.538 ). In the asymptomatic group, there were 3,2 , and 22 volunteers whose CL schedule was daily, biweekly, and monthly, respectively. In the symptomatic group, there were 6,3 , and 18 volunteers following the same CL schedules, respectively. In contrast, we found significant differences

1194 | www.corneajrnl.com
TABLE 1. Clinical Variables of the Groups Included at the Preliminary Visit

\begin{tabular}{|c|c|c|c|}
\hline Variables & $\begin{array}{c}\text { Asymptomatic } \\
\text { Group } \\
\text { Mean } \pm \text { SD } \\
\text { or Median } \\
\text { (Interquartile } \\
\text { Range) }\end{array}$ & $\begin{array}{c}\begin{array}{c}\text { Symptomatic } \\
\text { Group }\end{array} \\
\text { Mean } \pm \text { SD } \\
\text { or Median } \\
\text { (Interquartile } \\
\text { Range) }\end{array}$ & $\boldsymbol{P}$ \\
\hline $\begin{array}{l}\text { Visual acuity (logarithm of } \\
\text { the minimum angle of } \\
\text { resolution) }\end{array}$ & $-0.06 \pm 0.05$ & $-0.05 \pm 0.05$ & 0.45 \\
\hline BUT (s) & $6.5 \pm 3.5$ & $4.6 \pm 3.1$ & $0.03 *$ \\
\hline $\begin{array}{l}\text { Corneal staining (Oxford } \\
\text { scale) }\end{array}$ & $0(0-0)$ & $0(0-0)$ & 0.77 \\
\hline Schirmer I test (mm) & $24.6 \pm 10.6$ & $21.0 \pm 11.5$ & 0.28 \\
\hline
\end{tabular}

$(P=0.01)$ between groups for artificial tear use before the study; no participant from the asymptomatic group used them, whereas 7 out of 27 symptomatic wearers did.

The mean percentage of detection of each molecule (including visits 1-4, before and after environmental exposure) was as follows: EGF (95\%), IL-1RA (94\%), IL-1ß (50\%), IL-2 (56\%), IL-4 (49\%), IL-6 (71\%), IL-8 (95\%), TNF- $\alpha$ (53\%), MCP-1 (96\%), and MMP-9 (78\%). Each molecule concentration obtained during each visit is detailed in Supplemental Digital Content 1 (see Table, http://links.lww.com/ICO/A436).

\section{Effect of the "Environment"}

Analyzing the changes provoked by each condition, the standard condition exposure provoked a significant increase in the concentration of EGF $(0.36 ; 95 \% \mathrm{CI}, 0.08$ to 0.64$)$ and a decrease of IL-1 $\beta(-0.48 ; 95 \% \mathrm{CI},-0.84$ to -0.12$)$ and IL-2 $(-0.48 ; 95 \% \mathrm{CI},-0.87$ to -0.09$)$. A significant increase of IL-6 level $(0.35 ; 95 \% \mathrm{CI}, 0.09$ to 0.62$)$ was observed after the adverse condition exposure (Fig. 1). Comparing adverse and standard conditions, the adverse condition provoked significantly lower change than the standard condition for EGF $(P=0.02)$ and significantly higher for IL-1 $\beta(P=0.029)$, IL-2 $(P=0.016)$, IL-6 $(P=$ $0.045)$, and TNF- $\alpha(P=0.029)$ (Fig. 1).

The other molecules analyzed (IL-1RA, IL-4, IL-8, MCP-1, and MMP-9) were unaffected by the environment $(P>0.05)$.

\section{Effect of the "CL Type"}

Tear levels of IL-1 $\beta$ significantly decreased when wearing the comfilcon A CL $(-0.51 ; 95 \% \mathrm{CI},-0.88$ to $-0.15)$, whereas no significant change was reported with omafilcon A $(0.06 ; 95 \%$ CI, -0.23 to 0.35$)$ (Fig. 2). Moreover, the change of IL-1 $\beta$ was significantly different comparing both CLs $(P=0.014)$ (Fig. 2).

The CL type did not show any effect on the other tear molecules $(P>0.05)$ : EGF, IL-1RA, IL-2, IL-4, IL-6, IL-8, TNF- $\alpha$, MCP-1, and MMP-9.

Copyright (C) 2016 Wolters Kluwer Health, Inc. All rights reserved. 

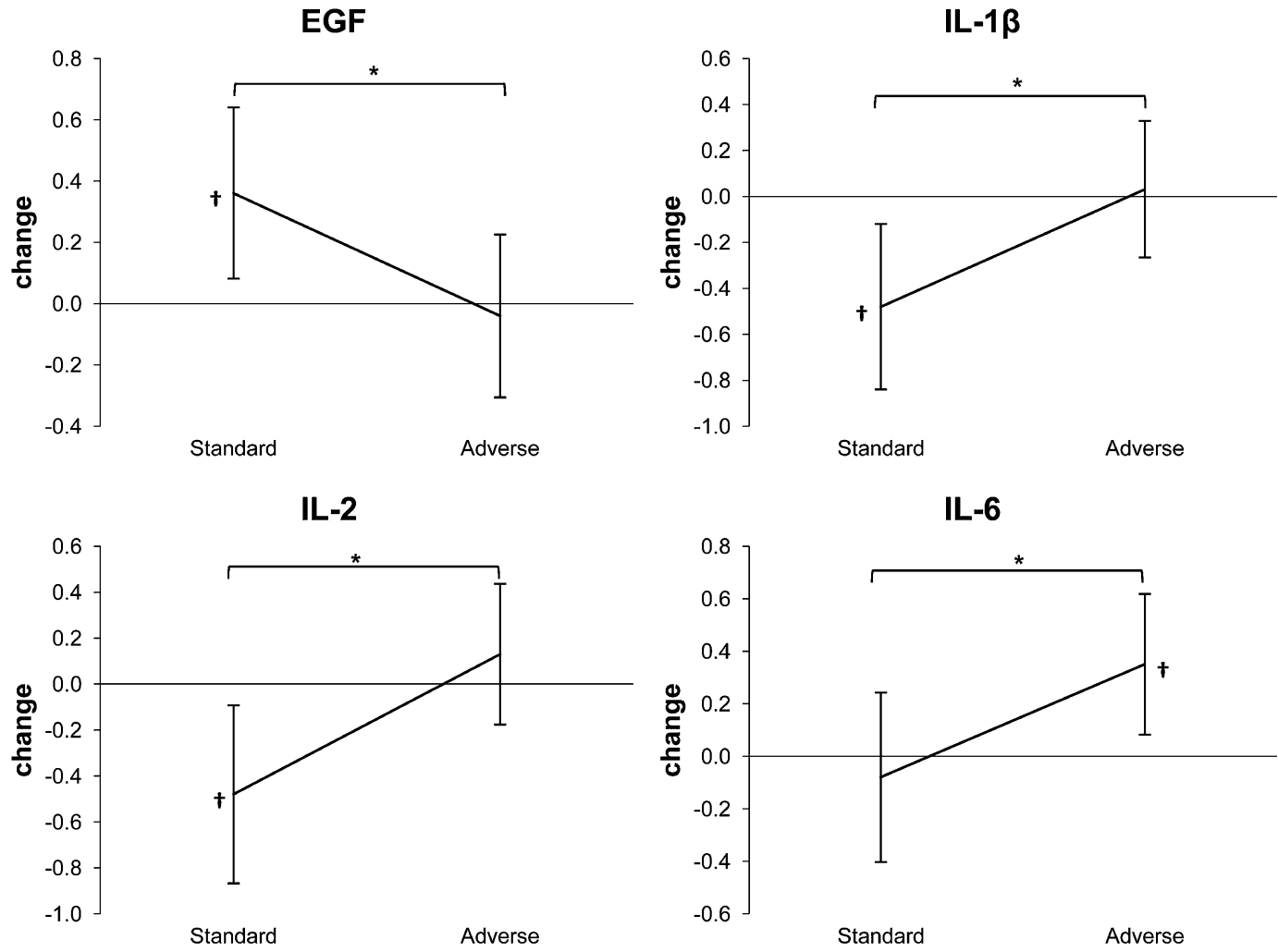

TNF- $\alpha$

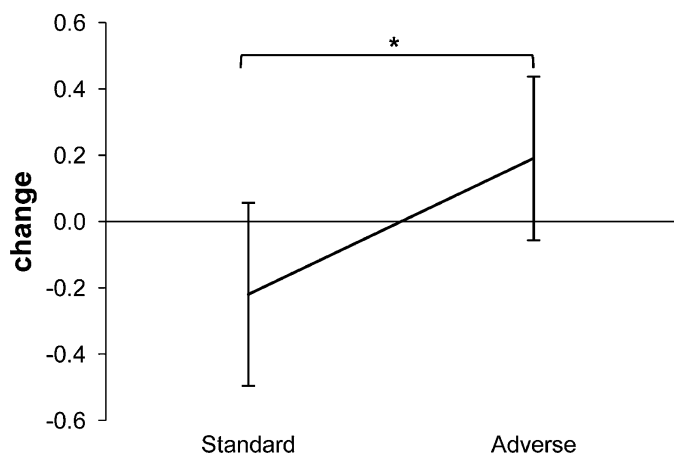

FIGURE 1. Environmentally produced changes in EGF, IL-1 $\beta, \mathrm{IL}-2, \mathrm{IL}-6$, and TNF- $\alpha$ concentrations. * Significant $(P<0.05)$ difference between the changes produced by the 2 environments. $\dagger$ Significant $(P<0.05)$ environmentally produced change in molecule concentration.

\section{Effect of the "Symptom-Based Grouping"}

No significant effects of the symptom-based grouping were found in any tear molecule analyzed $(P>0.05)$ : EGF, IL-1 $\beta$, IL1RA, IL-2, IL-4, IL-6, IL-8, TNF- $\alpha$, MCP-1, and MMP-9.

\section{Effect of the Interaction "Environment and CL Type"}

Under the standard condition, there was a significant decrease in IL-1 $\beta$ concentration when wearing comfilcon A CL $(-1.05 ; 95 \% \mathrm{CI},-1.56$ to -0.54$)$, but no change was observed with omafilcon A $(0.08 ; 95 \% \mathrm{CI},-0.40$ to 0.57$)$. These changes in IL-1 $\beta$ concentration were significantly different $(P=0.001)$ comparing both CLs (Fig. 3). No effects were found for this interaction under the adverse condition (Fig. 3).

No significant results were found for the remaining molecules $(P>0.05)$ : EGF, IL-1RA, IL-2, IL-4, IL-6, IL-8, TNF- $\alpha$, MCP-1, and MMP-9, although the change in MMP-9 showed a borderline significant effect $(P=0.055)$.

\section{Effect of the Interaction "Environment and Symptom-Based Grouping"}

No significant effects were found for this interaction in any tear molecule analyzed $(P>0.05)$ : EGF, IL-1 $\beta$, IL-1RA, IL-2, IL-4, IL-6, IL-8, TNF- $\alpha$, MCP-1, and MMP-9.

www.corneajrnl.com | 1195 


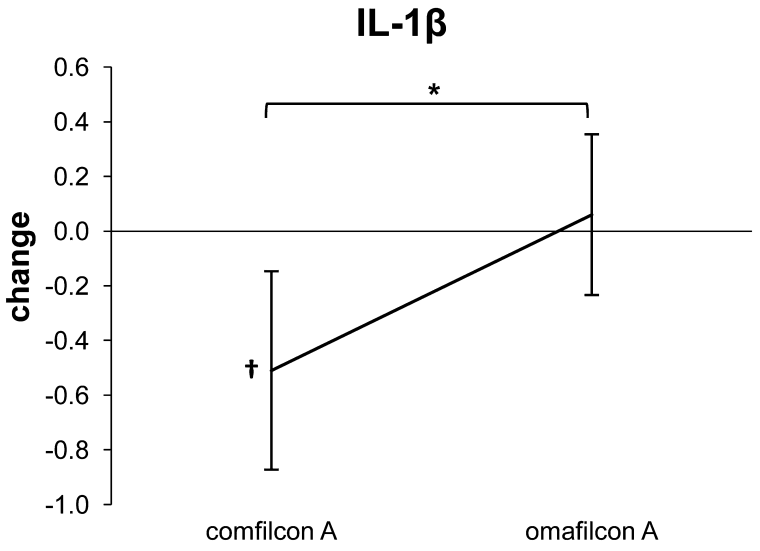

FIGURE 2. Changes produced in IL-1 $\beta$ concentration by $C L$ type. *Significant $(P<0.05)$ difference between the changes produced by the 2 lenses. †Significant $(P<$ 0.05 ) change produced by comfilcon $A$ in molecule concentration.

\section{Effect of the Interaction "CL Type and Symptom-Based Grouping"}

A significant decrease was found for asymptomatic subjects with comfilcon A for IL-1 $\beta(-0.90 ; 95 \%$ CI, -1.40 to $-0.40)$, but not with omafilcon A. A significant increase was found with omafilcon A for IL-6 $(0.50 ; 95 \% \mathrm{CI}, 0.17$ to 0.83$)$ and IL-8 $(0.46$; $95 \%$ CI, 0.07 to 0.86$)$, but not with comfilcon A (Fig. 4). Moreover, changes found for both CLs were significantly different for IL-1 $\beta(P=0.001)$, IL-6 $(P=0.040)$, and IL-8 $(P=0.016)$. No effects were found for this interaction in symptomatic subjects (Fig. 4).

The other tear molecules (EGF, IL-1RA, IL-2, IL-4, IL-6, IL-8, TNF- $\alpha$, MCP-1, and MMP-9) were not affected by this interaction $(P>0.05)$.

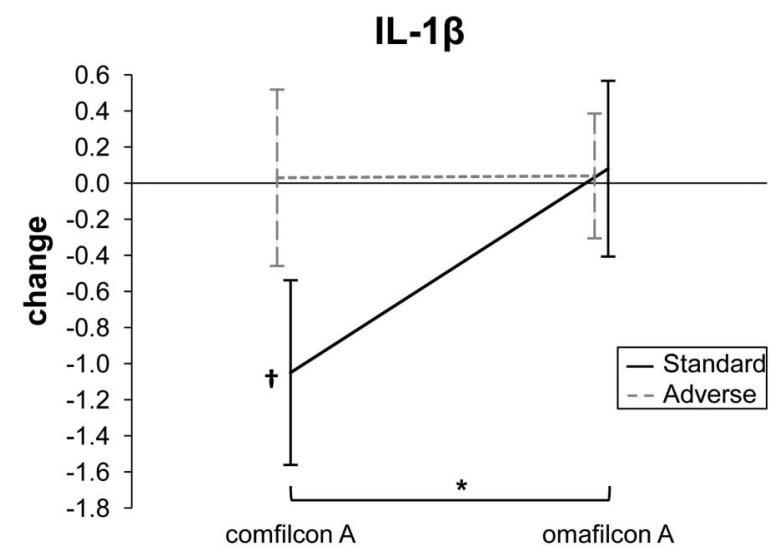

FIGURE 3. Changes in IL-1 $\beta$ concentration produced by the interaction between the environment and $\mathrm{CL}$ type. *Significant $(P<0.05)$ difference between the changes produced by the 2 lenses under the standard condition. †Significant $(P<0.05)$ change in molecule concentration produced by comfilcon $A$ under the standard condition.

1196 | www.corneajrnl.com

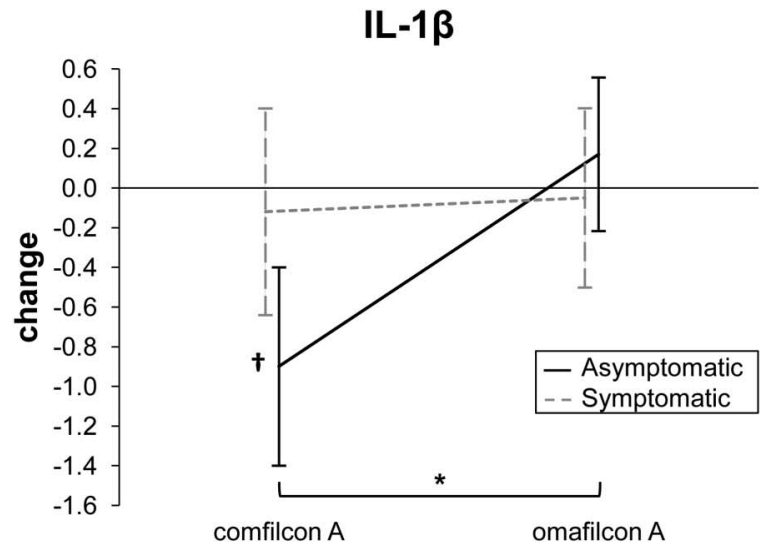

IL-6

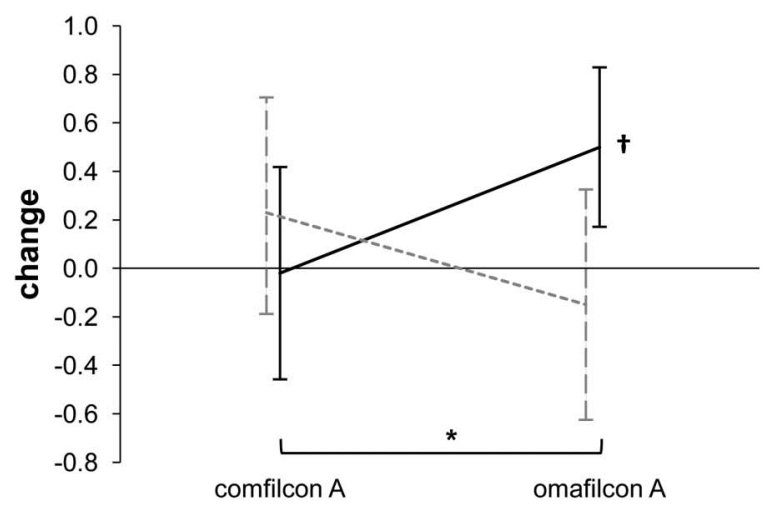

IL-8

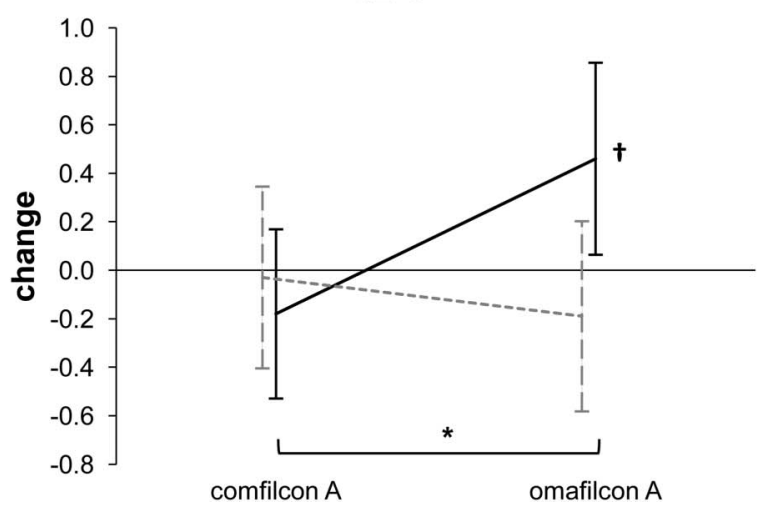

FIGURE 4. Changes in IL- $1 \beta, I L-6$, and IL- 8 concentrations produced by the interaction between $C L$ type and symptombased grouping. *Significant $(P<0.05)$ difference between the changes produced by the 2 lenses in the asymptomatic group. †Significant $(P<0.05)$ change produced by the $C L$ type in the molecule concentration.

\section{DISCUSSION}

In this study we have found that, after 2 hours of CL wear, an environmentally adverse condition provoked a more inflammatory profile (lower EGF and higher IL-1 $\beta$, IL-2, IL-6, and TNF- $\alpha$ concentrations) than a standard condition in $\mathrm{CL}$ wearers. Surprisingly, it is the standard condition that mainly affected the molecule levels, provoking an increase of

Copyright (C) 2016 Wolters Kluwer Health, Inc. All rights reserved. 
EGF and decrease of IL-1 $\beta$ and IL-2, whereas the adverse condition only provoked an increase of IL-6. In addition, there were also differences between CLs evaluated: patients wearing comfilcon A showed a decrease of IL-1 $\beta$ concentration, but this reduction was not seen with omafilcon A.

Our outcomes showed that the standard condition caused an effect that could be considered "protective" because EGF tear levels significantly increased but the concentration of inflammatory cytokines such as IL-1 $\beta$ and IL-2 significantly decreased. In contrast, this effect was not observed after our volunteers were exposed to the adverse condition. Instead, the changes in molecule concentrations constituted a "proinflammatory" scenario because IL-6 tear levels significantly increased. Besides, changes observed in EGF, IL-1 $\beta$, IL-2, IL-6, and TNF- $\alpha$ concentrations were significantly different from those observed after the standard condition, which further shows the "proinflammatory" effect of this desiccating condition simulating an air flight. All these results are in agreement with previous outcomes from our group where the effect of the environmental condition was studied in both healthy and dry eye patients. ${ }^{9}, 10$ These studies have shown that under an adverse condition (simulating an aircraft cabin), EGF concentration was significantly reduced and IL-6 tear levels were significantly increased in both healthy ${ }^{10}$ and dry eye patients, ${ }^{9}$ an effect that was not found under a standard condition either. ${ }^{9}$ Although we found no significant decrease in EGF concentration under the adverse condition, these levels were significantly lower than those found under the standard condition. These similarities between these studies ${ }^{9,10}$ and the findings in our CL users further confirm the potential of desiccating indoor environments to stimulate an inflammatory response not only in healthy subjects and dry eye patients but also in CL wearers.

Our results showed that the IL-1 $\beta$ tear concentration was significantly reduced after wearing the comfilcon $\mathrm{A}$ in comparison with the omafilcon $\mathrm{A}$, and also when wearing the comfilcon A under the standard condition in comparison with the other 3 situations. Despite the fact that no studies have yet detected IL-1 $\beta$ altered in CL wearers, it has been found to be increased in tears in dry eye patients, and even shows a positive correlation with dry eye syndrome severity. ${ }^{36}$ Thus, the decrease of IL-1 $\beta$ found might indicate that the use of comfilcon $\mathrm{A}$ under a standard condition may provide a less proinflammatory environment than the other situations. This result might be related to the higher oxygen permeability of comfilcom A (a silicone hydrogel CL), which in combination with a standard condition, produces a less proinflammatory effect on the ocular surface.

Similarly, the outcomes of the interaction between CL type and symptom-based grouping revealed that the IL-1 $\beta$, IL-6, and IL-8 tear concentrations were dependent on the CL type but only for asymptomatic subjects. IL- $1 \beta$ has been found elevated in tears of dry eye patients, ${ }^{36}$ IL- 6 has been shown to be a mediator of pain, ${ }^{37}$ and IL-8 can induce hyperalgesia ${ }^{38}$; thus, changes in their concentrations might be related to $\mathrm{CL}$ discomfort. Therefore, our results may indicate that in symptomatic subjects the effect of the CL material would be minor because the ocular surface is already altered, whereas in asymptomatic wearers, CLs can produce different inflammatory levels, depending on the CL type used.
This study has some limitations. First, only $2 \mathrm{CL}$ types were tested; other commercially available CL types could behave differently on the eye. Therefore, the results obtained are largely related to the CLs themselves and should be applied with caution to other CL designs and materials. Second, tear collection was always performed when the subjects were wearing CLs; consequently, our results describe the effect that the environmental conditions have on the ocular surface during CL wear. Therefore, as no tear collection was done without CL wear, the effect that these environmental conditions have on these subjects in the absence of CL wear was not studied and is unknown. And finally, CL wear has been found to decrease the tear film break-up time ${ }^{39}$ and the tear meniscus volume ${ }^{40}$ and to increase tear osmolarity. ${ }^{41}$ These findings might be associated with a reduction in the aqueous component of tears and could have an impact on the concentration of tear molecules (eg, cytokine levels) because of the change in the dilution rate, affecting our results. Further studies in CL wearers about the effect of environmental conditions including some other CL types and tear collection at different times and in the absence of CL wear are warranted.

In conclusion, the secretion of several tear inflammatory mediators during $\mathrm{CL}$ wear differs depending on the environmental conditions, standard or adverse, and the CL type, omafilcon A or comfilcon A. This might help to understand the effect of the environment and CL materials on the ocular surface of CL wearers. Differences observed between symptomatic and nonsymptomatic CL wearers will also provide insights for future strategies to overcome CL discomfort and inflammatory events in CL wearers.

\section{ACKNOWLEDGMENTS}

The authors thank Carmen García-Vázquez for technical assistance.

\section{REFERENCES}

1. National Research Council (US) Committee on Air Quality in Passenger Cabins of Commercial Aircraft. The Airliner Cabin Environment and the Health of Passengers and Crew. Washington, DC: National Academies Press (US); 2002.

2. González-García MJ, González-Sáiz A, de la Fuente B, et al. Exposure to a controlled adverse environment impairs the ocular surface of subjects with minimally symptomatic dry eye. Invest Ophthalmol Vis Sci. 2007; 48:4026-4032.

3. Nilsson SE, Andersson L. Contact lens wear in dry environments. Acta Ophthalmol (Copenh). 1986;64:221-225.

4. Morgan PB, Efron N. In vivo dehydration of silicone hydrogel contact lenses. Eye Contact Lens. 2003;29:173-176.

5. Maruyama K, Yokoi N, Takamata A, et al. Effect of environmental conditions on tear dynamics in soft contact lens wearers. Invest Ophthalmol Vis Sci. 2004;45:2563-2568.

6. Ousler GW III, Anderson RT, Osborn KE. The effect of senofilcon A contact lenses compared to habitual contact lenses on ocular discomfort during exposure to a controlled adverse environment. Curr Med Res Opin. 2008;24:335-341.

7. Kojima T, Matsumoto Y, Ibrahim OM, et al. Effect of controlled adverse chamber environment exposure on tear functions in silicon hydrogel and hydrogel soft contact lens wearers. Invest Ophthalmol Vis Sci. 2011;52: 8811-8817.

8. Galor A, Kumar N, Feuer W, et al. Environmental factors affect the risk of dry eye syndrome in a United States veteran population. Ophthalmology. 2014;121:972-973. 
9. Tesón M, González-García MJ, López-Miguel A, et al. Influence of a controlled environment simulating an in-flight airplane cabin on dry eye disease. Invest Ophthalmol Vis Sci. 2013;54:2093-2099.

10. López-Miguel A, Tesón M, Martín-Montañez V, et al. Dry eye exacerbation in patients exposed to desiccating stress under controlled environmental conditions. Am J Ophthalmol. 2014;157:788-798.

11. Wolkoff P. Ocular discomfort by environmental and personal risk factors altering the precorneal tear film. Toxicol Lett. 2010;199:203-212.

12. Glasson MJ, Stapleton F, Keay L, et al. The effect of short term contact lens wear on the tear film and ocular surface characteristics of tolerant and intolerant wearers. Cont Lens Anterior Eye. 2006;29:41-44.

13. Moschos MM, Eperon S, Guex-Crosier Y. Increased eotaxin in tears of patients wearing contact lenses. Cornea. 2004;23:771-775.

14. Philipp W, Speicher L, Humpel C. Expression of vascular endothelial growth factor and its receptors in inflamed and vascularized human corneas. Invest Ophthalmol Vis Sci. 2000;41:2514-2522.

15. Schultz CL, Kunert KS. Interleukin-6 levels in tears of contact lens wearers. J Interferon Cytokine Res. 2000;20:309-310.

16. Kallinikos P, Morgan P, Efron N. Assessment of stromal keratocytes and tear film inflammatory mediators during extended wear of contact lenses. Cornea. 2006;25:1-10.

17. Dogru M, Ward SK, Wakamatsu T, et al. The effects of 2 week senofilcon-A silicone hydrogel contact lens daily wear on tear functions and ocular surface health status. Cont Lens Anterior Eye. 2011;34:77-82.

18. Poyraz C, Irkec M, Mocan MC. Elevated tear interleukin-6 and interleukin-8 levels associated with silicone hydrogel and conventional hydrogel contact lens wear. Eye Contact Lens. 2012;38:146-149.

19. Yüksel Elgin C, İskeleli G, Talaz S, et al. Comparative analysis of tear film levels of inflammatory mediators in contact lens users. Curr Eye Res. 2015;16:1-7.

20. Craig JP, Willcox MD, Argüeso $P$, et al. The TFOS international workshop on contact lens discomfort: report of the contact lens interactions with the tear film subcommittee. Invest Ophthalmol Vis Sci. 2013;54:TFOS123-156.

21. Nichols KK, Redfern RL, Jacob JT, et al. The TFOS international workshop on contact lens discomfort: report of the definition and classification subcommittee. Invest Ophthalmol Vis Sci. 2013;54:TFOS14-19.

22. Berry M, Purslow C, Murphy PJ, et al. Contact lens materials, mucin fragmentation and relation to symptoms. Cornea. 2012;31:770-776.

23. Menzies KL, Jones L. In vitro analysis of the physical properties of contact lens blister pack solutions. Optom Vis Sci. 2011;88:493-501.

24. Federal Aviation Administration. Aeronautics and space. In: Code of Federal Aviation Regulations. Washington, DC: U.S Government Printing Office; 1996:1-59.
25. Norback D, Lindgren T, Wieslander G. Changes in ocular and nasal signs and symptoms among air crew in relation to air humidification on intercontinental flights. Scand J Work Environ Health. 2006;32:138-144.

26. Gallagher NM, Marienau KJ, Illig PA, et al. Conveyance and transportation issues. In: Gary W, ed. Brunette Health Information for International Travel. Philadelphia, PA: Elsevier, Inc; 2009:412-427.

27. Enríquez-de-Salamanca A, Castellanos E, Stern ME, et al. Tear cytokine and chemokine analysis and clinical correlations in evaporative-type dry eye disease. Mol Vis. 2010;16:862-873.

28. Helsel DR, Cohn TA. Estimation of descriptive statistics for multiplycensored water-quality data. Water Resour Res. 1988;24:1997-2004.

29. Lee L. NADA: Nondetects and Data Analysis for Environmental Data. $R$ Package Version 1.5-6. 2013. Available at: http://CRAN.R-project.org/ package $=$ NADA. Accessed February 20, 2015.

30. Lubin JH, Colt JS, Camann D, et al. Epidemiologic evaluation of measurement data in the presence of detection limits. Environ Health Perspect. 2004;112:1691-1696.

31. Friendly M. Power Analysis for ANOVA Designs. Available at: http:// www.math.yorku.ca/SCS/Online/power/. Accessed November 13, 2014.

32. Cohen J. Statistical Power Analysis of the Behavioural Sciences. 2nd ed. New York, NY: Academic Press; 1988.

33. Holm S. A simple sequentially rejective multiple test procedure. Scand $J$ Stat. 1979;6:65-70.

34. $\mathrm{R}$ Development Core Team. $R$ : A Language and Environment for Statistical Computing. Vienna, Austria: R Foundation for Statistical Computing; 2012. ISBN 3-900051-07-0.

35. Fox J, Weisberg S, ed. An $R$ Companion to Applied Regression. Thousand Oaks, CA: Sage; 2011.

36. Massingale ML, Li X, Vallabhajosyula M, et al. Analysis of inflammatory cytokines in the tears of dry eye patients. Cornea. 2009;28:1023-1027.

37. De Jongh RF, Vissers KC, Meert TF, et al. The role of interleukin-6 in nociception and pain. Anesth Analg. 2003;96:1096-1103.

38. Cunha FQ, Lorenzetti BB, Poole S, et al. Interleukin-8 as a mediator of sympathetic pain. Br J Pharmacol. 1991;104:765-767.

39. Hom MM, Bruce AS. Prelens tear stability: relationship to symptoms of dryness. Optometry. 2009;80:181-184.

40. Chen Q, Wang J, Shen M, et al. Tear menisci and ocular discomfort during daily contact lens wear in symptomatic wearers. Invest Ophthalmol Vis Sci. 2011;52:2175-2180.

41. Sarac O, Gurdal C, Bostanci-Ceran B, et al. Comparison of tear osmolarity and ocular comfort between daily disposable contact lenses: hilafilcon B hydrogel versus narafilcon A silicone hydrogel. Int Ophthalmol. 2012;32:229-233. 